\title{
Electron pair localization function: A practical tool to visualize electron localization in molecules from quantum Monte Carlo data
}

Cite as: J. Chem. Phys. 121, 1725 (2004); https://doi.org/10.1063/1.1765098

Submitted: 16 February 2004 . Accepted: 03 May 2004 . Published Online: 08 July 2004

Anthony Scemama, Patrick Chaquin, and Michel Caffarel

\section{ARTICLES YOU MAY BE INTERESTED IN}

A simple measure of electron localization in atomic and molecular systems

The Journal of Chemical Physics 92, 5397 (1990); https://doi.org/10.1063/1.458517

Density-functional thermochemistry. III. The role of exact exchange

The Journal of Chemical Physics 98, 5648 (1993); https://doi.org/10.1063/1.464913

Natural population analysis

The Journal of Chemical Physics 83, 735 (1985); https://doi.org/10.1063/1.449486

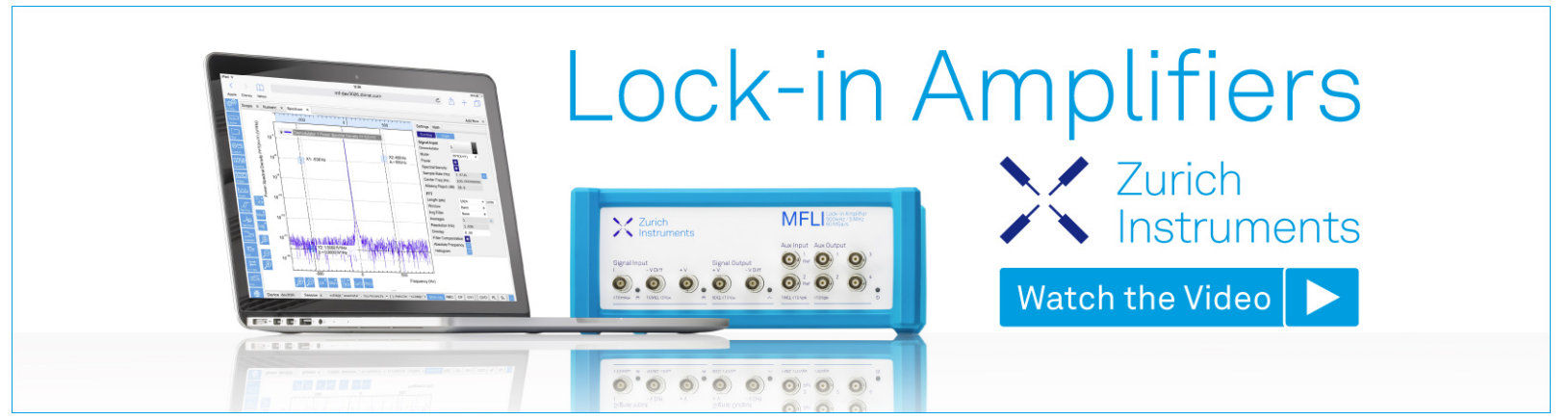

J. Chem. Phys. 121, 1725 (2004); https://doi.org/10.1063/1.1765098 


\title{
Electron pair localization function: A practical tool to visualize electron localization in molecules from quantum Monte Carlo data
}

\author{
Anthony Scemama, ${ }^{\text {a) }}$ Patrick Chaquin, and Michel Caffarel \\ Laboratoire de Chimie Théorique, UMR 7616 du CNRS, Université Pierre et Marie Curie Paris VI, \\ Case 137, 4, place Jussieu 75252 PARIS Cedex 05, France
}

(Received 16 February 2004; accepted 3 May 2004)

\begin{abstract}
In this work we introduce an electron localization function describing the pairing of electrons in a molecular system. This function, called "electron pair localization function," is constructed to be particularly simple to evaluate within a quantum Monte Carlo framework. Two major advantages of this function are the following: (i) the simplicity and generality of its definition; and (ii) the possibility of calculating it with quantum Monte Carlo at various levels of accuracy (Hartree-Fock, multiconfigurational wave functions, valence bond, density functional theory, variational Monte Carlo with explicitly correlated trial wave functions, fixed-node diffusion Monte Carlo, etc). A number of applications of the electron pair localization function to simple atomic and molecular systems are presented and systematic comparisons with the more standard electron localization function of Becke and Edgecombe are done. Results illustrate that the electron pair localization function is a simple and practical tool for visualizing electronic localization in molecular systems. (C) 2004 American Institute of Physics. [DOI: 10.1063/1.1765098]
\end{abstract}

\section{INTRODUCTION}

In recent years quantum Monte Carlo (QMC) approaches, a set of stochastic methods that solve exactly the Schrödinger equation, have been found very successful in calculating ground-state energies of various molecular systems, e.g., Refs. 1-6. The accuracy achieved is impressive: It is in general superior to that obtained with the most accurate approaches of standard computational chemistry [coupled cluster, multiconfigurational self-consisent field (MCSCF), etc.]. Furthermore, the number of electrons that can be treated is rather large. To give an example, let us mention the recent calculations of Williamson et al. for hydrogenated silicon clusters. ${ }^{7}$ Using linear-scaling techniques to reduce the computational cost of QMC algorithms, clusters including up to 984 valence electrons were shown to be accessible. Quite importantly, there is no practical limitation preventing such results from being extended to even greater systems in the near future.

Now, although ground-state energies are of primary interest, it is clear that a chemical understanding of a complex molecular system requires more than the knowledge of the sole ground-state energy. In general, chemists are interested in rationalizing and quantifying the structure and reactivity of the system in terms of various quantities related to electron localization. A number of important questions we wish to answer include, for example, the nature of the bonding between atoms, the localization of lone pairs, the local concentration or depletion of the charge, etc. In standard computational chemistry several approaches have been developed to analyze and visualize the electronic distribution in the ordinary three-dimensional (3D) space. Among them we can cite, e.g., the methods analyzing the deformation densi-

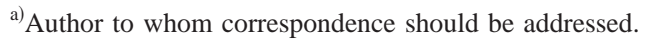

ties (a build-up of charge between two atoms is interpreted as the existence of a bond), ${ }^{8}$ the methods based on the topological analysis of the electron density or its Laplacian (see, Bader ${ }^{9}$ ), the methods studying the topography of the molecular electrostatic field, ${ }^{10}$ and, also, approaches using as indicator the electron localization function (ELF) describing the amount of local Pauli repulsion between electrons. ${ }^{11,12}$ Of course, this list cannot be considered as exhaustive since defining a successful qualitative model for the description of chemical structure is an everlasting theme in chemistry since the pioneering electron-pair model of Lewis.

In this work we propose to exploit the accurate data obtained in quantum Monte Carlo simulations to get some additional insights into the electron localization properties of molecules. To do that, we introduce a localization function describing the pairing of electrons in a molecular system. This function, called "electron-pair localization function" (EPLF), is built to be particularly easy to evaluate within a QMC framework. As illustrated here by several applications to simple atomic and molecular systems EPLF appears to be an effortless and practical tool for describing electronic features. As we shall see, the 3D-EPLF plots have some similarities with the plots obtained using the ELF of Becke and Edgecombe. ${ }^{11}$ However, there are also some important differences. These various points will be discussed below.

The organization of the paper is as follows: In Sec. II the basic features of QMC needed to understand the present work are briefly presented. In particular, we summarize the various probability densities produced by QMC. Section III is devoted to the definition and presentation of our EPLF. A brief presentation of the more standard ELF to which our results will be compared is also given. Section IV presents a number of practical details related to simulations. In Sec. V our results for several atoms and molecules $(\mathrm{Ne}, \mathrm{Ar}, \mathrm{LiH}$, $\mathrm{N}_{2}, \mathrm{CH}_{3}$, and $\mathrm{F}_{2}$ ) are presented. For the $\mathrm{F}_{2}$ case we give a 
detailed comparative study between the ELF and the EPLF analysis. Finally, in Sec. VI a summary of the main results of this work is presented.

\section{QUANTUM MONTE CARLO}

In a quantum Monte Carlo scheme a series of "states" or "configurations" are generated using some elementary stochastic rules. Here, a configuration is defined as the set of the $3 N$-electronic coordinates ( $N$ number of electrons), the positions of the nuclei being fixed (Born-Oppenheimer condition)

$$
\vec{R}=\left(\vec{r}_{1}, \ldots, \vec{r}_{N}\right) .
$$

Stated differently, a configuration $\vec{R}$ may be viewed as a "snapshot" of the molecule showing the instantaneous positions of each electron. Stochastic rules are chosen so that configurations are generated according to some target probability density, $\Pi(\vec{R})$. Note that the probability density is defined over the complete $3 N$-dimensional configuration space and not over the ordinary 3D space. Many variants of QMC can be found in the literature (referred to with various acronyms: VMC, DMC, PDMC, GFMC, etc). They essentially differ by the type of stochastic rules used and/or by the specific stationary density produced. In practice, the two most popular QMC approaches used for simulating complex molecular systems are the so-called variational Monte Carlo (VMC) and fixed-node diffusion Monte Carlo (FN-DMC) methods. Both methods will be employed here. Let us briefly summarize the basic features of these methods useful for the following (for a detailed presentation, see, e.g., Ref. 13).

\section{A. Variational Monte Carlo}

In a VMC calculation the probability density generated is given by

$$
\Pi_{\mathrm{VMC}}(\vec{R})=\psi_{T}^{2}(\vec{R}),
$$

where $\psi_{T}$ is a high-quality electronic trial wave function. A commonly used expression for $\psi_{T}$ consists of a product of two terms. The first term is standard and is introduced to describe the one-particle shell-structure of molecules. It is obtained from a preliminary Hartree-Fock (HF) or densityfunctional theory (DFT) ab initio calculation and is expressed as one (or a combination of a few) determinant(s) of single-particle orbitals. The second term is introduced to reproduce the electron-electron cusp condition of the exact wave function and, also, to incorporate some explicit coupling between electron-nucleus and electron-electron coordinates (see Ref. 14). Note that the electron-electron cusp condition is known to be particularly difficult to fulfill in standard ab initio calculations using expansions over oneelectron basis sets (necessity of considering very high values of the orbital momentum). The explicitly correlated term is usually referred to as the Jastrow factor. In a spin-free formalism our trial wave function is written as

$$
\psi_{T}(\vec{R})=D^{\uparrow}(\vec{R}) D^{\downarrow}(\vec{R}) \exp \left[\sum_{\alpha} \sum_{\langle i, j\rangle} U\left(r_{i \alpha}, r_{j \alpha}, r_{i j}\right)\right],
$$

where the sum over $\alpha$ denotes a sum over the nuclei, $\Sigma_{\langle i, j\rangle}$ a sum over the pair of electrons, and $D^{\sigma}(\sigma=\uparrow$ or $\downarrow)$ are determinants made of one-particle space orbitals. Different expressions for the Jastrow part have been presented in the literature. Here, we have chosen the following form: ${ }^{15}$

$$
\begin{aligned}
U\left(r_{i \alpha}, r_{j \alpha}, r_{i j}\right)= & s\left(x_{i j}\right)+p^{(\alpha)}\left(x_{i \alpha}\right)+c_{1} x_{i \alpha}^{2} x_{j \alpha}^{2} \\
& +c_{2}\left(x_{i \alpha}^{2}+x_{j \alpha}^{2}\right) x_{i j}^{2}+c_{3} x_{i j}^{2},
\end{aligned}
$$

with

$$
\begin{aligned}
& x_{i j}=\frac{r_{i j}}{1+b_{\sigma} r_{i j}}, \\
& x_{i \alpha}=\frac{r_{i \alpha}}{1+b_{\alpha} r_{i \alpha}}, \\
& s(x)=s_{1} x+s_{2} x^{2}+s_{3} x^{3}+s_{4} x^{4}, \\
& p^{(\alpha)}(x)=p_{1}^{(\alpha)} x+p_{2}^{(\alpha)} x^{2}+p_{3}^{(\alpha)} x^{3}+p_{4}^{(\alpha)} x^{4},
\end{aligned}
$$

$b_{\sigma}$ can take two different values depending on the spin of the pairs of electrons considered. In this latter expression the quantities $\left\{b_{\sigma}, b_{\alpha}, c_{i}, s_{i}, p_{i}^{(\alpha)}\right\}$ play the role of parameters.

The critical step in a VMC approach is the optimization of the parameters entering the trial wave function. A standard method consists in searching for parameters minimizing the fluctuations in configuration space of the local energy defined as

$$
E_{L}(\vec{R}) \equiv H \Psi_{T} / \Psi_{T} .
$$

This criterion is based on the fact that for the exact wave function the local energy reduces everywhere to a constantthe exact energy - and, thus, the fluctuations of the local energy entirely vanish. Accordingly, small fluctuations are associated with "good" trial wave functions. A number of methods have been developed to perform efficiently the optimization step within a QMC framework. In this work, we have used the correlated sampling method of Umrigar et al., ${ }^{16}$ an approach based on the minimization of the weighted variance of the local energy over a set of fixed configurations.

Once the optimal parameters have been determined, the quality of the resulting trial wave function is usually good: A major part of the dynamical correlation energy (Coulomb hole) is recovered and the gross features of the one-particle background are also correctly described via the determinantal part (nondynamical part). For most atoms it is possible to recover up to $80 \%-90 \%$ of the exact correlation energy; ${ }^{14}$ for molecules the domain of variation lies usually between $30 \%$ and $90 \%$ (see Table I for our calculations on $\mathrm{F}_{2}$ ).

The numerical method (stochastic rules) employed to generate the VMC density, Eq. (2), is standard. It is based on the use of a generalized Metropolis algorithm. Since this aspect is not important here we refer the interested reader to the relevant literature. ${ }^{13}$

\section{B. Diffusion Monte Carlo}

In a diffusion Monte Carlo scheme the stochastic rules employed are the same as in the VMC case (Metropolis algorithm) plus a new rule corresponding to a branching (or 
TABLE I. Comparison of energies obtained for $\mathrm{F}_{2}$ at different levels of computation in atomic units. The interatomic distance is the experimental equilibrium distance, $R=2.668$. Statistical uncertainty on the last digit given in parenthesis.

\begin{tabular}{llc}
\hline \multicolumn{1}{c}{ Method } & \multicolumn{1}{c}{ Energy } & Correlation energy $(\%)$ \\
\hline HF/cc-pVTZ & -198.752055 & \\
HF exact & $-198.7741^{\mathrm{a}}$ & $0 \%$ \\
CISD/cc-pVTZ & -199.263205 & $65 \%$ \\
CCSD(T)/cc-pVTZ & -199.320514 & $72 \%$ \\
VMC & $-199.163(3)$ & $51(0.4) \%$ \\
DMC & $-199.439(9)$ & $88(1.2) \%$ \\
Expt. & $-199.530^{\mathrm{b}}$ & $100 \%$ \\
\hline \hline
\end{tabular}

${ }^{a}$ Reference 31.

${ }^{b}$ Estimate of the exact nonrelativistic energy. Obtained by correcting the experimental value of the JANAF tables (Ref. 32) with the ZPE and spinorbit contributions (Ref. 33).

birth-death) process. More precisely, depending on the magnitude of the local energy a given configuration can be destroyed (when the local energy is greater than some estimate of the exact energy) or duplicated a certain number of times (local energy lower than the exact energy). It can be shown that the stationary density resulting from these rules is now given by

$$
\Pi_{\mathrm{DMC}}(\vec{R})=\psi_{T}(\vec{R}) \phi_{0}(\vec{R}),
$$

where $\phi_{0}(\vec{R})$ denotes the ground-state wave function. Actually, because the density $\Pi_{\text {DMC }}$ is necessarily positive (as any stationary density resulting from some stochastic rules), $\phi_{0}$ is not exactly the exact ground-state wave function but some approximate one resulting from the additional constraint that $\phi_{0}$ must have the same sign as the trial wave function (so that the product in Eq. (6) always remains positive). Such a constraint implies that the nodes (points where the wave function vanishes) are identical for $\psi_{T}$ and $\phi_{0}$. The resulting error is called the "fixed-node" error. Generally, this approximation is very good (see Table I), the fixed-node error on total energies represents usually a small fraction of the total correlation energy (this error depends only on the quality of the nodes, see, e.g., the discussion in Ref. 17).

\section{C. “Exact” quantum Monte Carlo}

Of course, beyond VMC, Eq. (2), and fixed-node DMC densities, Eq. (6) we would be interested in generating the exact density associated with the unknown ground-state wave function, $\phi_{0}$

$$
\Pi_{\text {exact }}(\vec{R})=\phi_{0}^{2}(\vec{R}) .
$$

In principle, obtaining such a density within a quantum Monte Carlo approach is possible. This can be done by resorting to one of the variants of the so-called "forward walking" schemes. ${ }^{18-20}$ Unfortunately, in practice, such schemes are known to be intrinsically unstable and, therefore, very time consuming for large systems. For applications on complex systems the possibility of getting a stable density of configurations is unrealistic.

\section{THE ELECTRON PAIR LOCALIZATION FUNCTION}

Before presenting the electron pair localization function let us first present briefly the ELF introduced by Becke and Edgecombe. ${ }^{11}$

\section{A. ELF}

The ELF is designed to probe the amount of electronic localization in the usual 3D space. For the sake of simplicity, ELF is constructed to take its values within the interval $[0,1]$. The upper limit, $\mathrm{ELF}=1$, corresponds to perfect localization, the value $E L F=1 / 2$ corresponds to a level of localization comparable to that of an uniform electron gas, and small values of ELF correspond to delocalization. The fundamental quantity from which ELF is built is the parallel-spin conditional pair probability density, $P_{\text {cond }}^{\sigma \sigma}\left(\vec{r}_{1}, \vec{r}_{2}\right)$, a quantity which gives the probability of finding a $\sigma$-spin electron at position $\vec{r}_{2}$ when a $\sigma$-spin electron is located at position $\vec{r}_{1}$. ELF is defined as

$$
\operatorname{ELF}(\vec{r})=\frac{1}{1+\chi_{\sigma}^{2}(\vec{r})},
$$

with

$$
\chi_{\sigma}(\vec{r})=\frac{D_{\sigma}(\vec{r})}{D_{\sigma}^{0}(\vec{r})},
$$

and where the local function $D_{\sigma}(\vec{r})$ is the leading term of the Taylor expansion of the spherically averaged conditional pair probability $P_{\text {cond }}^{\sigma \sigma}\left(\vec{r}_{1}, \vec{r}_{2}\right)$ (point 1 is considered as the reference point $\vec{r}$ and the conditional pair probability is integrated with respect to $\vec{r}_{2}$ over the sphere of radius $s$ and centered at $\left.\vec{r}=\vec{r}_{1}\right)$ :

$$
P_{\text {cond }}^{\sigma \sigma}(\vec{r}, s)=\frac{1}{3} D_{\sigma}(\vec{r}) s^{2}+\ldots .
$$

In Eq. (9) the function $D_{\sigma}^{0}$ is introduced as a reference quantity: It corresponds to the value of the function $D_{\sigma}$ for the uniform electron gas at the same density $\rho_{\sigma}$.

For an independent-particle model, $D_{\sigma}(\vec{r})$ can be easily computed from the set of occupied orbitals $\psi_{i}$ as follows:

$$
D_{\sigma}(\vec{r})=\sum_{i}^{\sigma}\left|\vec{\nabla} \psi_{i}\right|^{2}-\frac{1}{4} \frac{\left|\vec{\nabla} \rho_{\sigma}\right|^{2}}{\rho_{\sigma}},
$$

and the reference quantity $D_{\sigma}^{0}$ is usually taken as that of the uniform noninteracting electron gas with density $\rho_{\sigma}$ as done in the original work of Becke and Edgecombe:

$$
D_{\sigma}^{0}(\vec{r})=\frac{3}{5}\left(6 \pi^{2}\right)^{2 / 3} \rho_{\sigma}(\vec{r})^{5 / 3} \text {. }
$$

Beyond independent-particle models described by a single determinant wave function the situation regarding ELF is somewhat confusing. In that case, several proposals for both the definition of ELF and/or its interpretation have been presented in the literature (however, all these interpretations become equivalent for independent particle systems). Here, it is not necessary to enter into such details (for that, see, e.g., Refs. 21 and 22). However, it is important to emphasize that there is no consensus on what definition and interpretation should be used for a general correlated manybody wave function. 


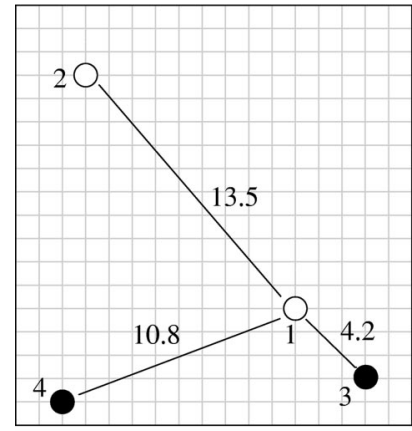

$\mathrm{EPLF}=0.52($ Electron 1$)$

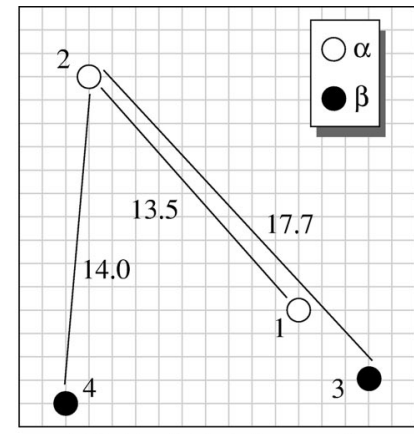

$\mathrm{EPLF}=-0.02($ Electron 2)
FIG. 1. Illustration of the EPLF for one configuration. On the left, the EPLF is computed for the elementary volume containing electron 1: $d_{\uparrow \uparrow}\left(\mathbf{r}_{1}\right)$ $=13.45, d_{\uparrow \downarrow}\left(\mathbf{r}_{1}\right)=4.24, \operatorname{EPLF}\left(\mathbf{r}_{1}\right)=(13.45-4.24) /(13.45+4.24)=0.52$. On the right, the EPLF is computed for the elementary volume containing electron 2: $\quad d_{\uparrow \uparrow}\left(\mathbf{r}_{2}\right)=13.45, \quad d_{\uparrow \downarrow}\left(\mathbf{r}_{2}\right)=14.03, \quad \operatorname{EPLF} \quad\left(\mathbf{r}_{2}\right)=(14.03$ $-13.45) /(14.03+13.45)=-0.02$.

\section{B. EPLF}

In this section we present the EPLF. The choice of its particular form is guided by the following remarks. First, we have chosen to adopt some important characteristics of the ELF approach: The EPLF is chosen to be local, bounded above and below and easy to calculate. Second, we have chosen to focus explicitly on the localization of pairs of electrons. Clearly, it is a most valuable point since pairs of electrons play a central role in our everyday interpretation of chemical structure and reactivity (Lewis model, VSEPR). The framework proposed to calculate such a localization function is that of quantum Monte Carlo approaches. As emphasized in the introduction, QMC are techniques of a great versatility and, therefore, the definition of the EPLF proposed below will be of practical use for any type of wave functions (HF, post-HF, valence bond, etc) and any level of computation (VMC, FN-DMC, "exact"). Many different localization functions could be chosen, we have found that the following form is particularly simple and efficient.

First, we introduce the two local quantities $d_{\sigma \sigma}(\vec{r})$ and $d_{\sigma \bar{\sigma}}(\vec{r})$ defined as follows:

$$
\begin{aligned}
& d_{\sigma \sigma}(\vec{r}) \equiv \sum_{i=1}^{N}\left\langle\left\langle\delta\left(\vec{r}-\vec{r}_{i}\right) \min _{j ; \sigma_{j}=\sigma_{i}}\left|\vec{r}_{i}-\vec{r}_{j}\right|\right\rangle\right\rangle, \\
& d_{\sigma \bar{\sigma}}(\vec{r}) \equiv \sum_{i=1}^{N}\left\langle\left\langle\delta\left(\vec{r}-\vec{r}_{i}\right) \min _{j ; \sigma_{j} \neq \sigma_{i}}\left|\vec{r}_{i}-\vec{r}_{j}\right|\right\rangle\right\rangle,
\end{aligned}
$$

where $\left\{\vec{r}_{k}\right\}_{k=1, N}$ are the positions of the $N$ electrons for a given configuration $\vec{R}, \sigma_{i}$ is the spin of the $i$ th electron $\left(\sigma_{i}\right.$ $=\uparrow, \downarrow)$, and $\langle\langle\ldots\rangle\rangle$ the stochastic average over the Monte Carlo configurations. As seen from these definitions $d_{\sigma \sigma}(\vec{r})$ [respectively, $\left.d_{\sigma \bar{\sigma}}(\vec{r})\right]$ is the average distance between an electron located at $\vec{r}$ and the closest spinlike (respectively, spin-unlike) electron of the molecule.

Now, we propose to define the EPLF as follows:

$$
\operatorname{EPLF}(\vec{r})=\frac{d_{\sigma \sigma}(\vec{r})-d_{\sigma \bar{\sigma}}(\vec{r})}{d_{\sigma \sigma}(\vec{r})+d_{\sigma \bar{\sigma}}(\vec{r})} .
$$

Figure 1 gives a simple pictorial representation of the con- struction of the EPLF in the case of only one configuration and four electrons in 2D.

By definition the EPLF takes its values within the interval $[-1,1]$. It gives a local indicator of pairing as follows. In regions of space where electrons are unpaired the average distances between spinlike and spin-unlike electrons are similar, $d_{\sigma \bar{\sigma}} \approx d_{\sigma \sigma}$, and EPLF goes to zero. When spinunlike electrons are paired we have $d_{\sigma \bar{\sigma}} \ll d_{\sigma \sigma}$ and EPLF goes to 1 . Finally, when spinlike electrons are paired, $d_{\sigma \bar{\sigma}}$ $\gg d_{\sigma \sigma}$ and, thus, EPLF goes to -1 . Remark that to be defined the EPLF requires at least one pair of spinlike and one pair of spin-unlike electrons in the system. Note also that the definition of EPLF is particularly well suited to QMC; formula (13) can indeed be easily computed with any QMC scheme.

\section{COMPUTATIONAL DETAILS}

Trial wave functions. For all systems presented below, the Hartree-Fock wave functions have been computed at the B3LYP equilibrium geometry (except for $F_{2}$ for which we have used the experimental distance) using the GAMESS (Ref. 23) program with Dunning's correlation consistent double- $\zeta$ basis set. ${ }^{24}$ These wave functions are used to represent the determinantal part of the trial functions used in the quantum Monte Carlo computations. To remove the large statistical fluctuations associated with the wrong description of the wave function at the nuclei (the exact electron-nucleus cusp cannot be reproduced with finite Gaussian basis sets), the Gaussian-type core molecular orbitals have been replaced by the Slater-type atomic core orbitals given by Koga et al. ${ }^{25}$

QMC data. The densities relative to HF wave functions, Eq. (2), have been sampled by VMC and are labeled HFVMC. For several systems, the trial wave function has been improved by introducing an explicitly correlated Jastrow factor as described above, Eq. (4). The density corresponding to this wave function has been sampled by VMC, labeled JastVMC, and also by fixed-node diffusion Monte Carlo, labeled FN-DMC. For each molecule considered, a typical simulation includes a set of 800 independent walkers and a number of Monte Carlo steps per walker ranging from 10 thousands to 100 thousands. During each simulation we also take advantage of the symmetry of the molecules by applying symmetry operations on configurations (e.g., for a homonuclear diatomic molecule symmetrizing the configurations with respect to the $x, y$, and $z$ planes increase the number of configurations by a factor eight).

EPLF data. The continuous 3D space is represented using a $80 \times 80 \times 80$ three-dimensional grid. The EPLF is calculated as follows: For each Monte Carlo configuration generated the positions of the electrons are scanned, the elementary cubic volume of the 3D grid occupied by each electron is determined and the minimum distances appearing in the definition of EPLF are calculated. The noise in the localization function due to the statistical character of QMC simulations has been reduced by using a median blur filter which operates as follows: Each value of the unregularized function $\operatorname{EPLF}_{0}(\vec{r})$ is considered as being at the center of a $0.4 \times 0.4 \times 0.4 \AA$ larger box (containing several elementary volumes of the $3 \mathrm{D}$ grid). The list of values of the EPLF 
within this box are sorted, and the middle value of the list is affected to $\operatorname{EPLF}(\vec{r})$. This filter is commonly used in image manipulation applications when the noise is mostly impulsional. Compared to the more standard Gaussian blur filter (use of Gaussian functions instead of localized $\delta$ functions), the median blur filter reduces less the noise, but it modifies less the regions where the gradient is large. This latter point is particularly important here since we are interested in altering the contours of the localization function as less as possible.

ELF data. To compare the results of our pair localization function with Becke and Edgecombe's ELF, we have performed a number of Hartree-Fock and B3LYP calculations. The program used is GAUSSIAN 98 (Ref. 26) and the basis set employed is the Dunning's correlation-consistent polarized valence double-zeta basis set (cc-pVDZ) basis set. All calculations have been done at the molecular geometry used in QMC simulations. From these wave functions, the ELF has been computed with the TopMod series of programs. ${ }^{27}$

\section{EXAMPLES}

The following examples have been chosen to illustrate EPLF in various elementary situations. Systematic comparisons with ELF are given. First, we present two examples of rare gas atoms to illustrate how the atomic shell structure is recovered with EPLF. Then, several diatomic molecules are treated: The LiH molecule is chosen as an example of molecule having a large ionic character, the $\mathrm{N}_{2}$ molecule illustrates a strongly covalent triple bond with lone pairs, and the $\mathrm{CH}_{3}$ radical a case where an unpaired electron is present. Finally, we present a more detailed analysis of the molecule $\mathrm{F}_{2}$, a system for which the predictions of ELF and EPLF are qualitatively different.

\section{A. Shell structure of atoms}

In Figs. 2 and 3 we present a number of 1D plots for the $\mathrm{Ne}$ and Ar atoms, respectively. In the upper part the standard radial density distributions $r^{2} \rho(r)$ are shown. For neon, the density is computed with VMC using either a Hartree-Fock wave function (solid line) or a correlated wave function (broken line). In the latter case the amount of correlation energy recovered is about $85 \%$. As seen on Fig. 2 the difference in radial density resulting from the explicitly correlated part is small and does not change the overall shape of the curve. The shell structure of Ne (two shells corresponding to the principal quantum numbers $n=1$ and $n=2$ ) is clearly seen. The location of the minimum between the two shells is about $r \sim 0.3$ a.u. while the two maxima are located at $r$ $\sim 0.12$ a.u. $(n=1)$ and $r \sim 0.6$ a.u. $(n=2)$. For argon, we also get the usual shell structure consisting of three shells $(n=1,2,3)$. The maxima are located at $r \sim 0.05$ a.u. $(n$ $=1), r \sim 0.31$ a.u. $(n=2)$, and $r \sim 1.2$ a.u. $(n=3)$, the two minima being at $r \sim 0.12$ a.u. $(n=1-2)$ and $r \sim 0.80$ a.u. $(n$ $=2-3$ ).

In the lower part of Figs. 2 and 3 the ELF and EPLF are presented. As already known ${ }^{11}$ the atomic shell structure is correctly reproduced with ELF. For the neon atom the two shells are centered at $r=0(n=1)$ and $r \sim 0.8$ a.u. $(n=2)$, while the minimum between shells is at $r \sim 0.3$ a.u. $(n$
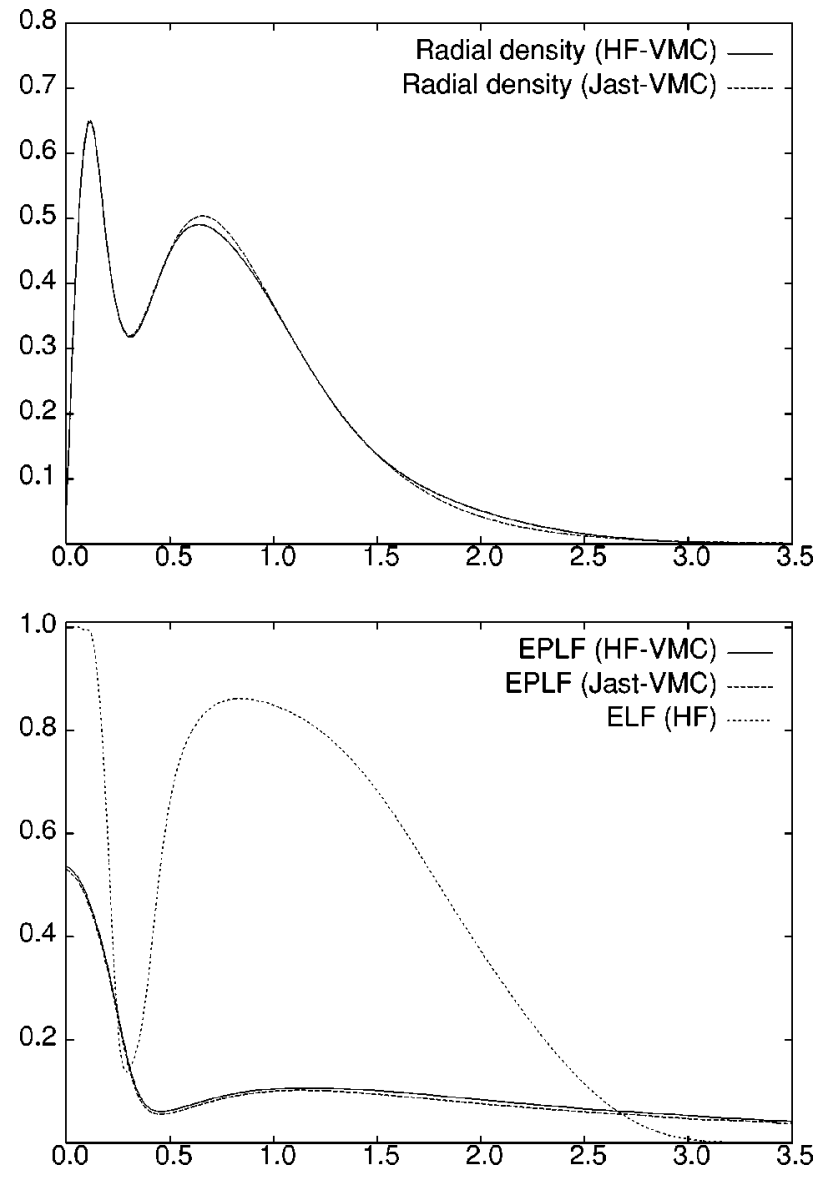

FIG. 2. ELF and EPLF for Ne.

$=1-2$ ). These values are rather close to those obtained for the radial density. For the argon atom, the corresponding values are $r=0(n=1), \quad r \sim 0.35$ a.u. $(n=2), \quad$ and $r$ $\sim 1.45$ a.u. $(n=3)$ for the maxima, and $r \sim 0.15$ a.u. ( $n$ $=1-2)$ and $r \sim 0.75$ a.u. $(n=2-3)$ for the minima. Here also, the values of the minima and maxima given by ELF are close to the those obtained from the radial density. Note also that the values of the maxima of ELF corresponding to each shell are not very different and all close to one (1 and 0.86 for neon; $1,0.87$, and 0.88 for argon). According to ELF, this result is interpreted as the fact that the degree of electronic localization in the various regions of important charge density is similar.

Let us now turn our attention to the EPLF data. Similarly to the ELF or the radial density, we see that the EPLF reveals the atomic shell structure: The two and three maxima of neon and argon are recovered. In addition to this we also have a similar structure for the inner doubly occupied $1 s$ shell. It is described by a rather high and narrow peak located at the origin. Note that the domain covered by this peak is similar to what we get with ELF or the radial density. A large value for the EPLF can be easily interpreted as follows. Close to the nucleus there are two spin-unlike electrons occupying the $n=1$ shell and, for each of these electrons, the closest spinlike electron necessarily occupies the $n=2$ shell. Accordingly, we have $d_{\sigma \sigma} \gg d_{\sigma \bar{\sigma}}$ and, therefore, a large value of EPLF. Using this argument we can compute an estimate of the EPLF value. Taking $d_{\sigma \sigma}$ equal to the maximum distance between two electrons sitting on two spheres corresponding 

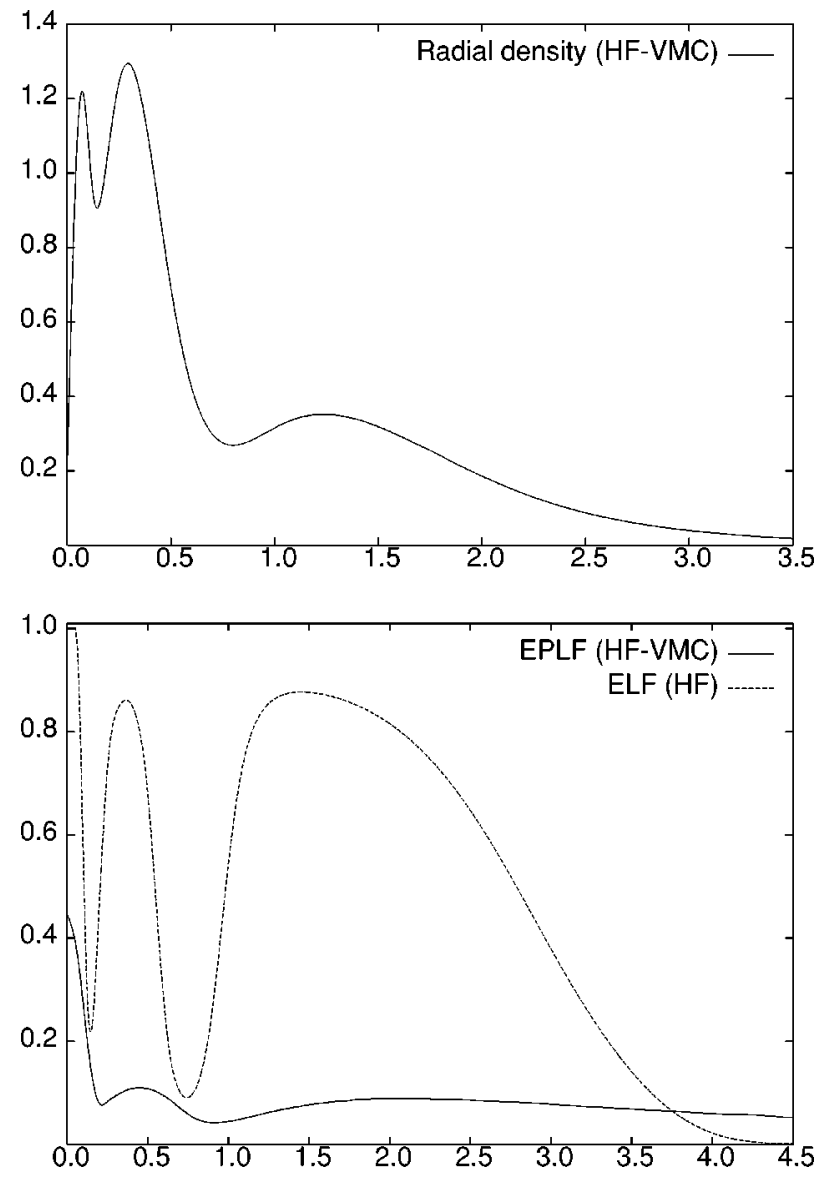

FIG. 3. ELF and EPLF for Ar.

to the $n=1$ and $n=2$ shells (electrons are repulsing each other and are supposed to minimize their repulsion) and $d_{\sigma \bar{\sigma}}$ the maximum distance between two electrons sitting on the same sphere corresponding to the $n=1$ shell, we get EPLF $\sim 0.5$ for neon and argon. This value is in good agreement with the EPLF values at the origin $(0.54$ for neon and 0.45 for argon). The shape of the outer shells $(n=2,3)$ is quite different from what we get with ELF or the radial distribution. In the valence domain the EPLF has the shape of a very broad and flat peak. The maximum value is quite small and is close to the value corresponding to the limit of infinite distances. For the $n=1$ maximum, the ratio of the EPLF values at the maximum and at $r \sim \infty$ is about 13 for neon and 9 for argon. For $n=2$ this ratio falls down to about 2 for both neon and argon. Finally, in the case of the $n=3$ shell of argon, the ratio is only 1.7. Such results are interesting since small values of EPLF are directly associated with similar average distances between spinlike and spin-unlike electrons. As a consequence, it seems not appropriate to visualize electrons in the various atomic shells as a set of tightly bounded pairs but better as a rather uniform fluid of electrons with comparable interelectronic distances.

\section{B. Ionic molecule: LiH}

The ELF and EPLF plots are displayed in Fig. 4. Both functions present two maxima localized at the nuclei. This result is expected since the $\mathrm{LiH}$ molecule having a strong

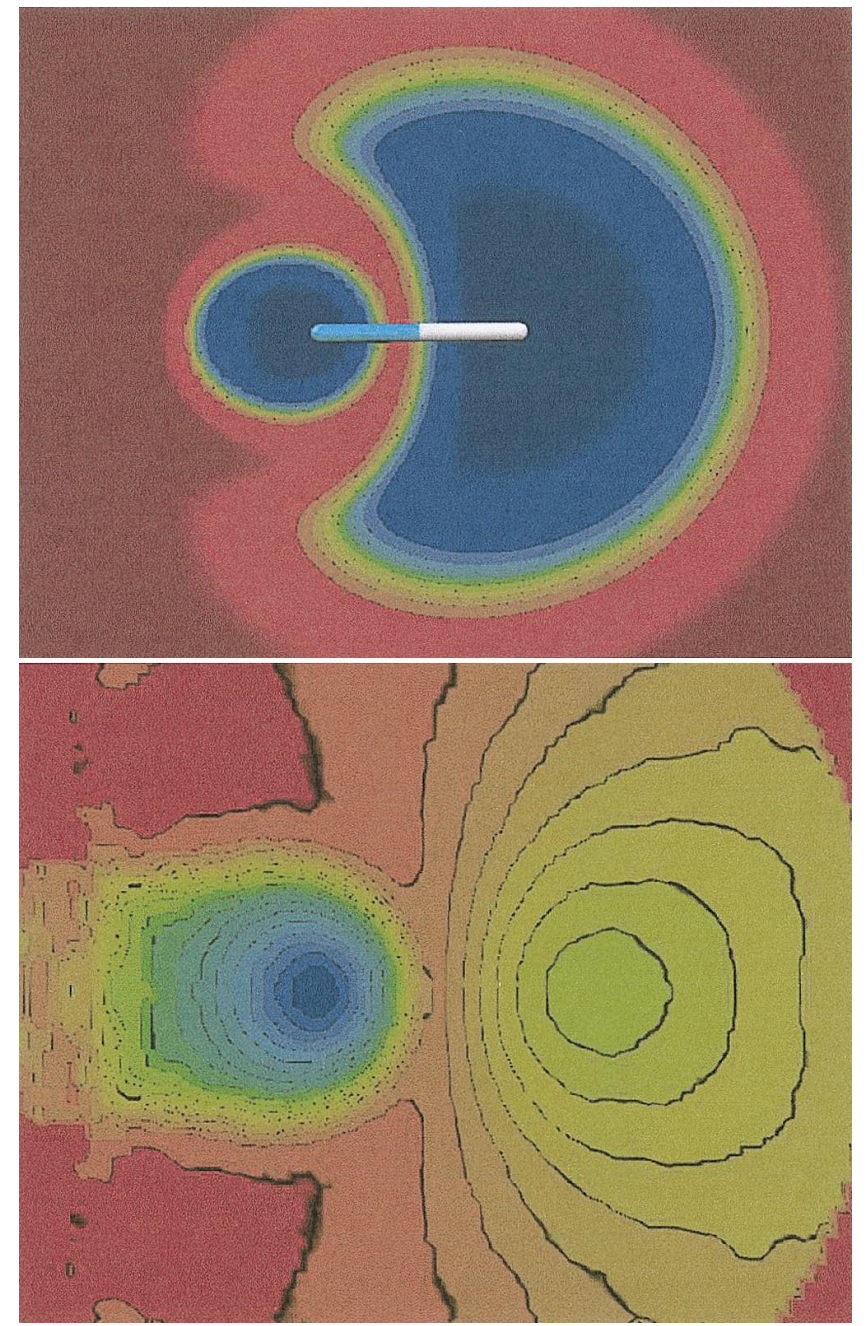

FIG. 4. (Color) ELF and EPLF for LiH in a molecular plane. Li atom on the left side, $\mathrm{H}$ on the right side. The contour maps were created using the MOLEKEL program. ${ }^{34}$ Low values appear in red and high values appear in blue.

ionic character it is usually described as consisting of two pairs of electrons, one centered on the $\mathrm{Li}$ nucleus $\left(\mathrm{Li}^{+}\right.$contribution) and the other one centered on the hydrogen $\left(\mathrm{H}^{-}\right.$ part). To better visualize these two maxima we also present the one-dimensional cut of both functions along the internuclear axis, Fig. 5. In our plots the lithium atom is located at the origin (left part of both figures) while the hydrogen atom is on the right hand side. As seen, the extent of ELF and EPLF are rather similar. However, the shapes of the functions are different. In contrast with ELF, the EPLF illustrates the difference of pairing associated with each pair. The average distance between the two electrons of the $\mathrm{Li}^{+} 1 s$ pair is small (large value of EPLF) while the electron pair around the hydrogen nucleus is much less confined (the EPLF peak is smaller and broader).

\section{Triple bond and lone pairs: $\mathbf{N}_{\mathbf{2}}$}

The ELF and EPLF plots are displayed in Fig. 6. Both functions present two maxima localized at the atomic cores and two maxima localized on the lone pairs. The ELF presents a single maximum in the region where a triple bond is 


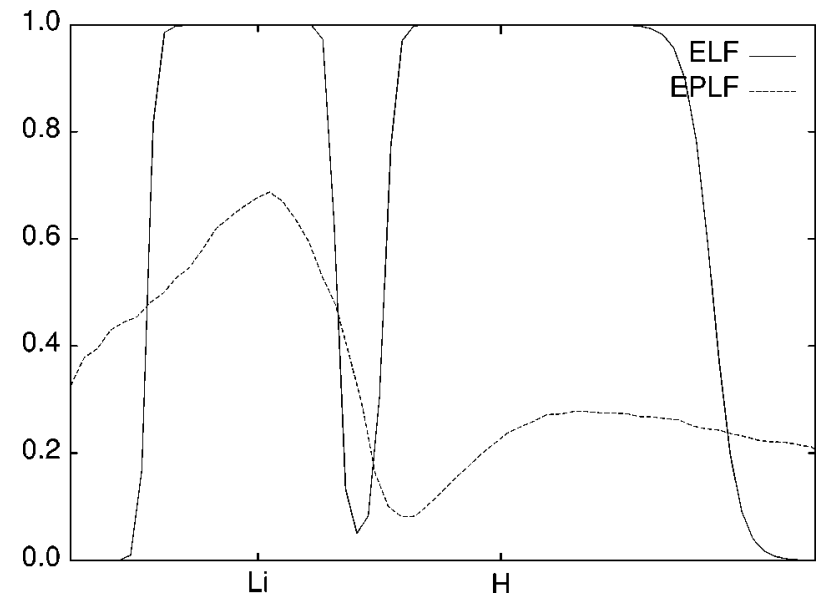

FIG. 5. ELF and EPLF for $\mathrm{LiH}$ along the $\mathrm{Li}-\mathrm{H}$ axis. $\mathrm{Li}$ on the left, $\mathrm{H}$ on the right.
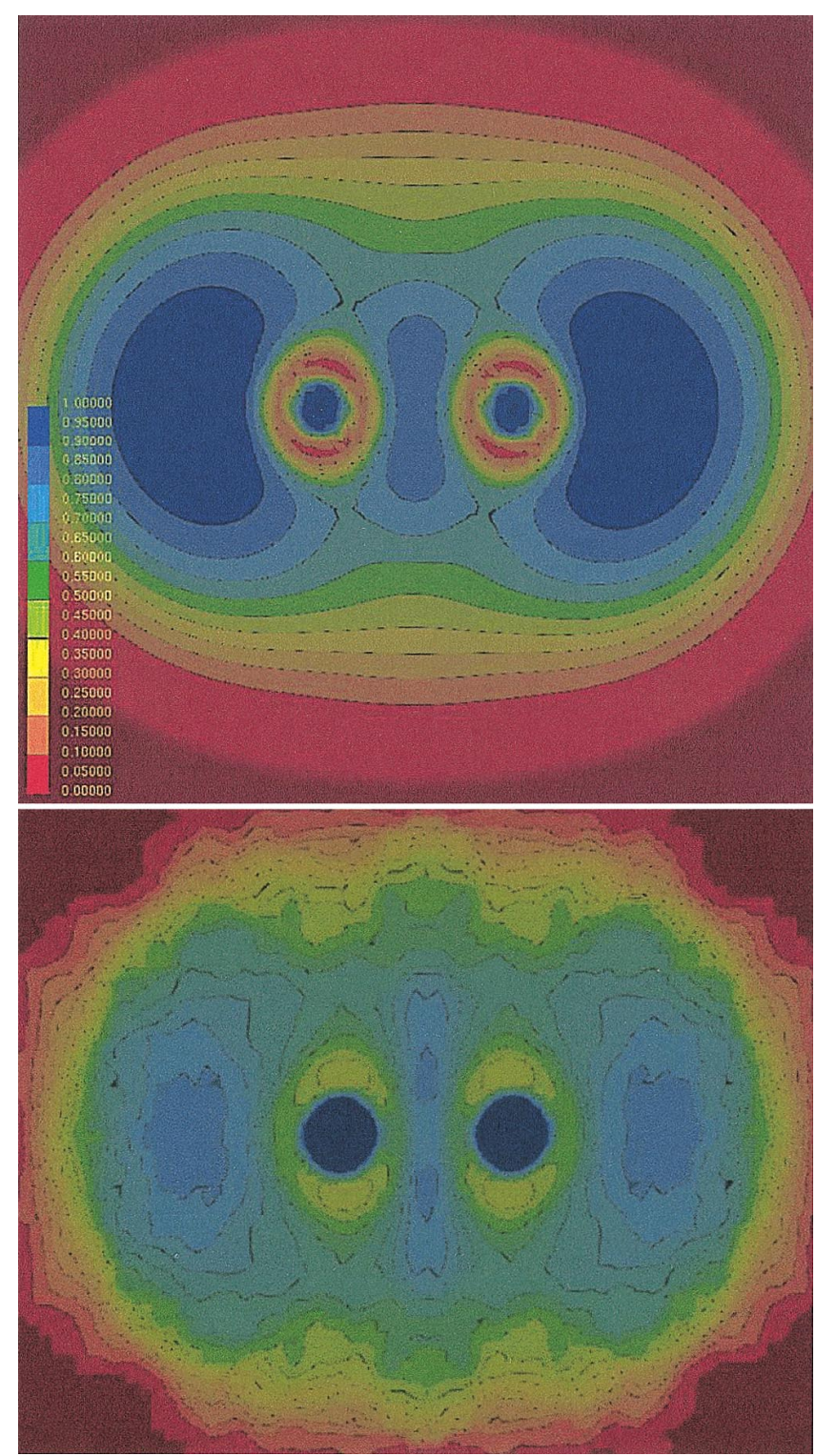

FIG. 6. (Color) ELF and EPLF for $\mathrm{N}_{2}$ in a molecular plane. Low values appear in red and high values appear in blue.
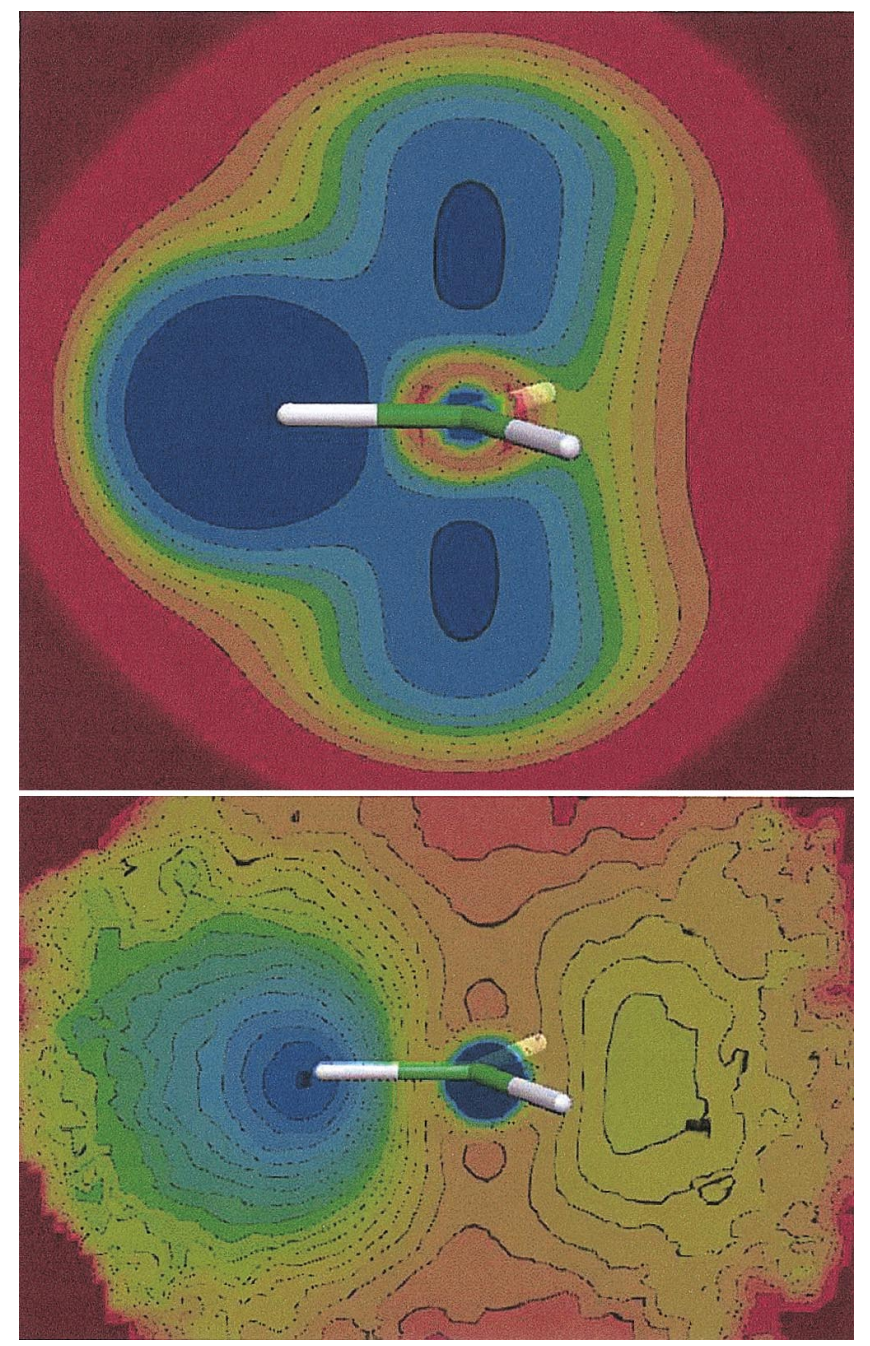

FIG. 7. (Color) ELF and EPLF for $\mathrm{CH}_{3}$ in a plane containing the carbon atom (central atom) and one hydrogen atom. The two other hydrogen atoms are symmetrically located with respect to the plane (back and front). Low values appear in red and high values appear in blue.

expected (Lewis representation). In contrast, the EPLF presents an infinity of degenerate maxima placed on a torus in the $\sigma_{h}$ plane of symmetry.

\section{Radical: $\mathrm{CH}_{3}$}

The ELF and EPLF plots are displayed in Fig. 7. As expected both functions present a maximum localized at the carbon core and three maxima localized on the hydrogen atoms. In contrast, ELF and EPLF give a different picture of the presence of a localized single electron. According to the very definition of ELF, the localized electron leads to the existence of a maximum. With EPLF, the situation is rather different: A single electron implies no pairing at all and, therefore, a small value of the function is obtained. Remark that the possibility of differentiating localized pairs and single electrons in such a way is an attractive feature of EPLF with respect to ELF. In Fig. 8 it is shown how the nonbonding unpaired electron can be localized by plotting an isosurface for a very small value of the EPLF. 


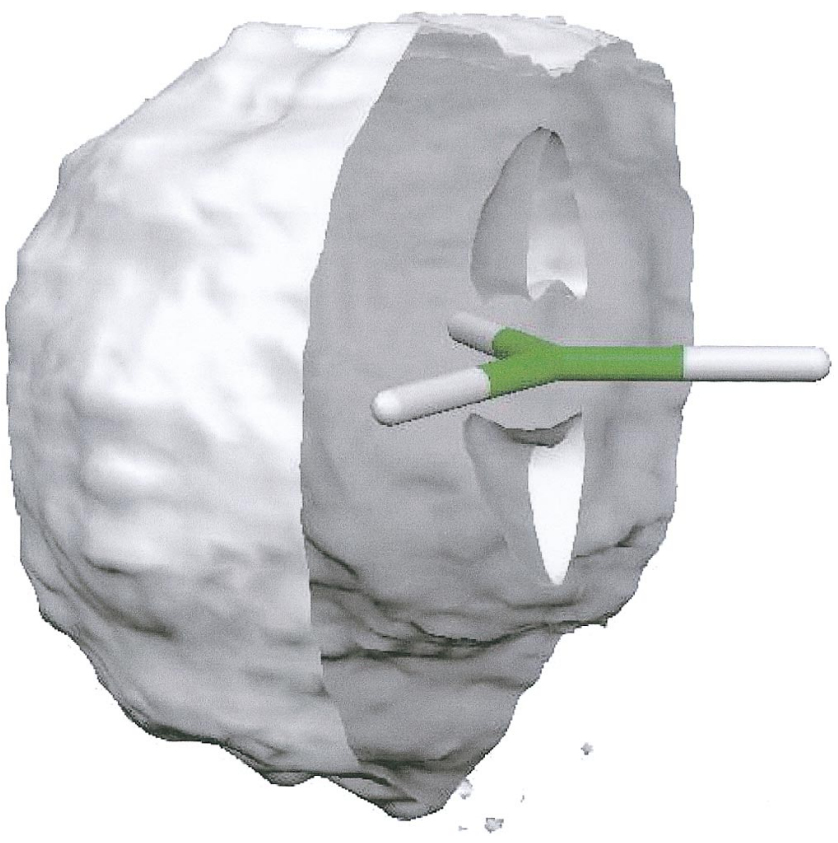

FIG. 8. (Color) Isosurface EPLF=0.04 for $\mathrm{CH}_{3}$.

\section{E. Covalent versus ionic bonding: $F_{2}$}

\section{ELF analysis}

Figure 9 presents the ELF plots obtained for the $\mathrm{F}_{2}$ molecule at the Hartree-Fock (upper part) and B3LYP (lower part) levels. The interatomic distance chosen is the experimental value, $R=2.668$ a.u. In both plots similar features are observed: Presence of maxima associated with core electron pairs and presence of degenerate maxima corresponding to lone pairs on a torus around each nucleus. However, there is one striking difference within the bonding region. At the Hartree-Fock level, ELF displays a maximum at the middle of the F-F bond. In contrast, at the B3LYP level, the same region displays two maxima on the F-F axis slightly shifted away symmetrically from the center towards the nuclei and a minimum along the axis at the symmetry center (a saddle point in the full space). To shed some light on the origin of this intriguing change of topology, we have recomputed ELF for different basis sets and methods (Hartree-Fock in small and very large basis sets, DFT at various levels: LDA, BP86, BLYP, GGA,...). Results show that the single maximum observed at the Hartree-Fock level turns out to result from the merging of two close peaks. Depending on conditions, these two peaks can appear either as two well-separated peaks (two maxima and a minimum in between along the internuclear axis) or one single peak (a maximum). Note that the possibility of the existence of such a structural instability of the critical points with respect to basis sets and correlation effects has already been emphasized in previous works (e.g., Refs. 21 and 28). It is thus not correct to attribute to much significance to the change of topology observed from HF to B3LYP and a more detailed analysis of ELF is called for. Fortunately, such an analysis has recently been presented by Llusar and collaborators ${ }^{28}$ for a number of systems including $F_{2}$. In their study the authors have followed the evolution under electron correlation of the "valence basin" populations
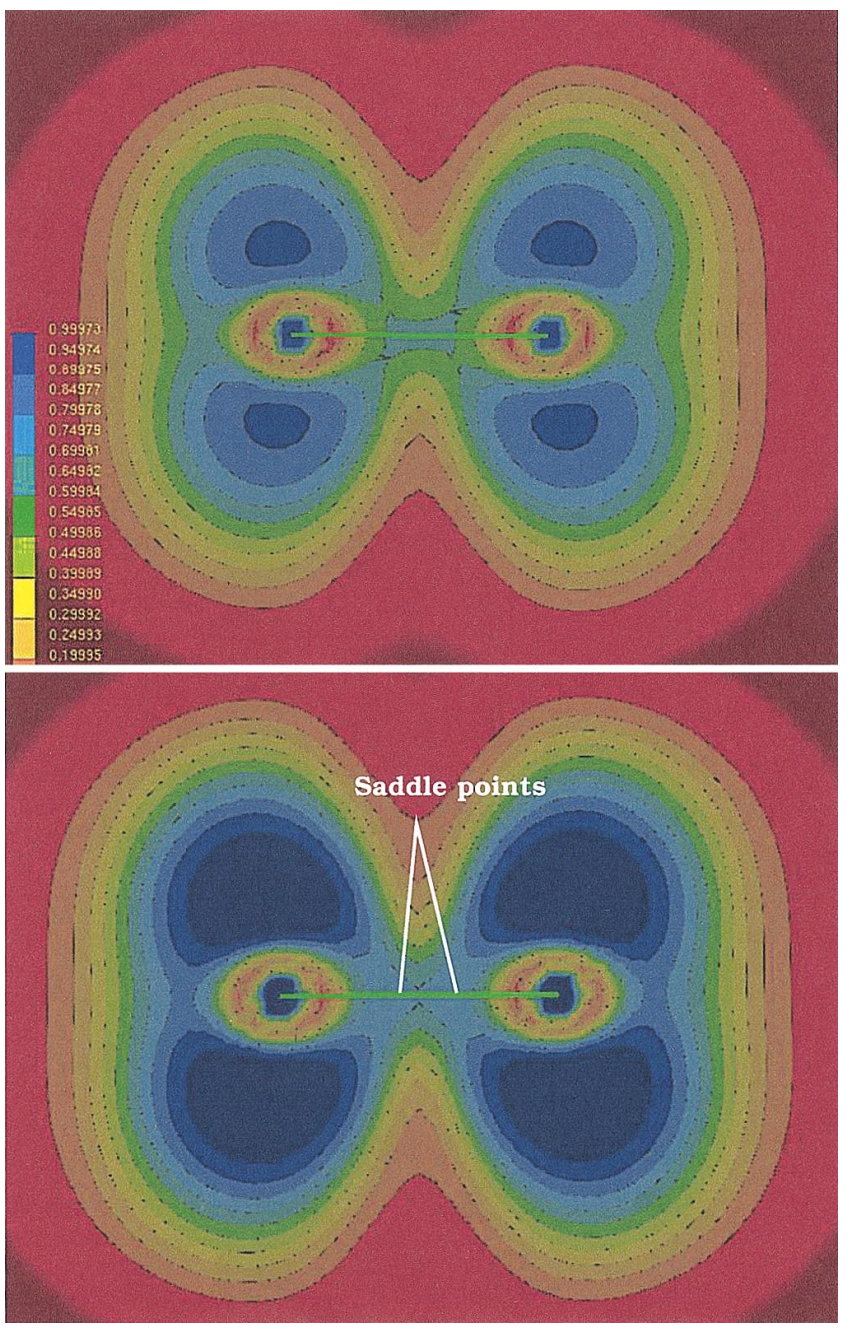

FIG. 9. (Color) ELF for $\mathrm{F}_{2}$ in a molecular plane, computed from a HartreeFock wave function (up) and from a B3LYP calculation (down). Low values appear in red and high values appear in blue.

(lone pair and "covalent" valence basins) associated with the attractors of ELF (such a study is based on a proposal of Silvi and Savin ${ }^{12}$ ). From their data they conclude that the topology of the ELF gradient dynamical system of $\mathrm{F}_{2}$ around the equilibrium distance is indeed structurally unstable and that the role of electron correlation consists essentially in decreasing the population of the valence basin responsible for bonding toward the atomic lone pairs. Furthermore, in contrast with most covalent bonds where this scenario occurs at large internuclear distances, for $\mathrm{F}_{2}$ it occurs at a rather short distance close to the equilibrium geometry and, thus, leads to some peculiar features. However, the amount of electron localization between the atoms remaining not negligible both at the HF and DFT levels (sufficiently large basis sets are to be used), it seems justified to conclude that the covalent character of $F_{2}$ is not negligible.

\section{EPLF analysis}

The EPLF has been computed at the HF-VMC, JastVMC and fixed-node DMC levels. To give an idea of the accuracy achieved we present in Table I the total energies corresponding to different levels of calculations. As seen in 


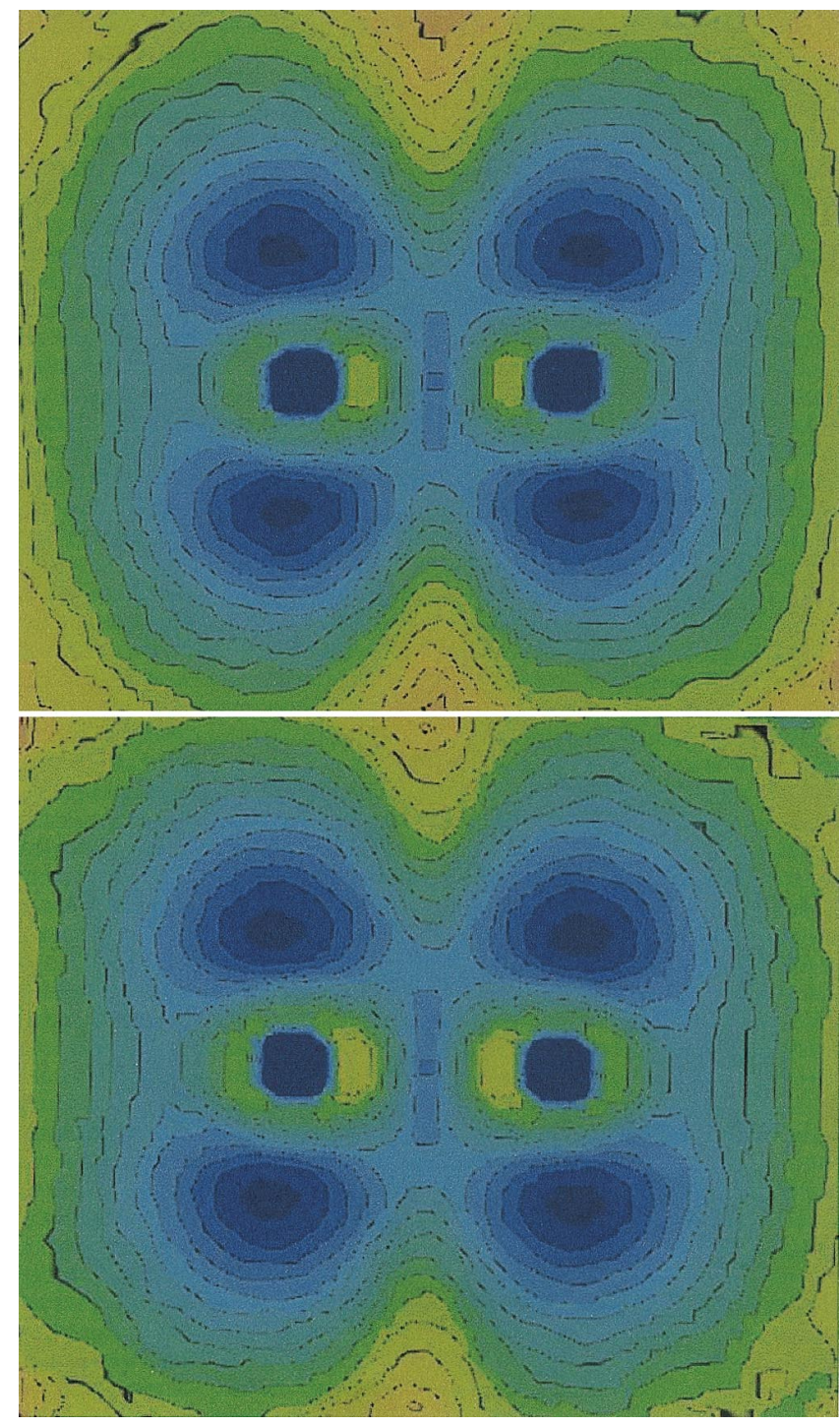

FIG. 10. (Color) EPLF for $\mathrm{F}_{2}$ along the F-F axis, computed from the HFVMC density (up) and from the Jast-VMC density (down). Low values appear in red and high values appear in blue.

the table the Jastrow wave function is rather accurate since $51 \pm 0.4 \%$ of the correlation energy is recovered. At the DMC level we recover up to $88 \pm 1.2 \%$. The EPLF plots obtained from VMC and fixed-node DMC calculations have been found to be very similar. Therefore, to avoid to duplicate data, we only present the Jast-VMC results. EPLF plots computed from the HF-VMC and Jast-VMC densities are presented in Fig. 10. In contrast with ELF we do not observe a change of topology when passing from the Hartree-Fock to the (highly) correlated levels. In both cases, we get the same set of critical points: A maximum in each core regions, an infinity of degenerate maxima corresponding to the lone pairs on a torus around each nucleus and, most importantly, a maximum at the symmetry center of the molecule.

To validate the presence of a maximum at the middle of the bond we have computed the EPLF for three hypothetical models consisting of noninteracting fluorine atoms (or ions), the interatomic distance being fixed at the equilibrium distance of the true interacting $\mathrm{F}_{2}$ molecule. The first model consists of the neutral system $\mathrm{F}_{\uparrow}+\mathrm{F}_{\downarrow}$, the second one de- scribes the pure ionic system $\mathrm{F}_{\uparrow \downarrow}^{-} \mathrm{F}^{+}$and, the third one, the resonant structure expressed as $\mathrm{F}_{\uparrow \downarrow}^{-} \mathrm{F}^{+} \leftrightarrow \mathrm{F}^{+} \mathrm{F}_{\uparrow \downarrow}^{-}$.

$E P L F$ for $F_{\uparrow}+F_{\downarrow}$. The wave function used for each atom is a standard Hartree-Fock determinant with a $p_{z}$ singly occupied orbital ( $z$ being the internuclear axis). One of the atoms is chosen with a $\alpha$ unpaired electron, the second one with a $\beta$ unpaired electron. To compute the EPLF for this noninteracting molecule, we have sampled the density of one single fluorine atom located at the position of the first atom of the hypothetical molecule (simulation 1). Next, we have moved the atom to the position of the second atom, flipped the spin of the single electron, and, then, sampled the second density (simulation 2). Both runs were made with identical random numbers, time steps, numbers of walkers, and total number of steps. The configurations saved during the two runs were merged: Each configuration of the noninteracting system is the combination of two configurations, one from simulation $\mathbf{1}$ and the other one from simulation 2 . The important result is that we did not observe any maximum between the two fluorine atoms of this fictitious system, but instead a minimum. Now, because the probability of finding a $p_{z}$ electron of one atom in a given region is independent on the position of the electrons of the other atom, we are in a case where the two $p_{z}$ electrons of opposite spin are unpaired. Accordingly, the presence of a minimum in the noninteracting system confirms that EPLF measures directly the electron pairing and that there is no influence of the oneelectron probability density. We conclude that the maximum observed for the interacting system is not an artifact resulting from the overlap of the electronic clouds of the two neutral atoms.

EPLF for $F_{\uparrow \downarrow}^{-} F^{+}$. Using the same strategy we have computed the EPLF in the case of the noninteracting system $\mathrm{F}^{-}+\mathrm{F}^{+}$. The EPLF of the $\mathrm{F}^{-}$anion has one maximum at the position of the nucleus, and an infinity of maxima on a sphere corresponding to the valence shell. For the $\mathrm{F}^{+}$cation (with a vacant $p_{z}$ orbital), an infinity of degenerate maxima on a torus in the $(x, y)$ plane are obtained. When the two noninteracting ions are fixed at the equilibrium distance of $\mathrm{F}_{2}$, we observe a saddle point between the two ions. This critical point, which results essentially from the interaction between the maxima associated with the $p_{x}$ and $p_{y}$ lone pairs of $\mathrm{F}^{+}$and the maxima of the $\mathrm{F}^{-}$valence shell, is found to be closer to $\mathrm{F}^{-}$than $\mathrm{F}^{+}$.

EPLF for $F_{\uparrow \downarrow}^{-} F^{+} \leftrightarrow F^{+} F_{\uparrow \downarrow}^{-}$. The computation of the EPLF has been done by applying to the configurations obtained in the previous case the mirror symmetry with respect to the plane perpendicular to the $\mathrm{F}^{+}-\mathrm{F}^{-}$axis and located at the middle of the bond. Configurations resulting from this operation are interpreted as describing the resonance between the two ionic structures: $\mathrm{F}^{+}-\mathrm{F}^{-} \leftrightarrow \mathrm{F}^{-}-\mathrm{F}^{+}$. Here also, we observe a saddle point at the symmetry center of the molecule.

It is important to emphasize that none of the three fictitious systems just presented exhibits a maximum of the EPLF at bond midpoint. Accordingly, it seems reasonable to conclude that the maximum observed in the true $\mathrm{F}_{2}$ molecule is not an artifact and is really due to the covalent contribution of the $\mathrm{F}_{2}$ bond. The computation of electron correlation ef- 
fects, even at the highest level of correlation (fixed-node DMC) does not eliminate this maximum, so the covalent character of $F_{2}$ is truly important (as opposed, for example, to $\mathrm{LiH}$ ). Note that such a result is in agreement with previous works based on high-quality valence bond calculations (see, e.g., Hiberty and collaborators, ${ }^{29,30}$ ).

Finally, let us mention that the present analysis based on all-electron calculations and similar analysis for other systems are not expected to change when pseudopotentials are used to describe cores. In the case of $F_{2}$, we have indeed verified that identical EPLF features in the valence region are obtained with and without pseudopotentials. The only difference found between both calculations is the existence of a minimum at the position of the nuclei when pseudopotentials are used.

\section{SUMMARY}

In this paper we have introduced an EPLF whose main feature is to reveal the differences in the average distances between spinlike and spin-unlike electrons. In regions where localized pairs of electrons are present (lone pairs, atomic pairs, bonds) the EPLF takes large values and displays maxima. In contrast, in regions where electrons behave essentially as an homogeneous fluid (spinlike and spin-unlike electrons being mixed together), the EPLF takes much smaller values. The form of the EPLF is simple, Eqs. (13) and (14), and has been chosen to be easily computable using quantum Monte Carlo. A major advantage of QMC is the possibility of evaluating the EPLF at various levels of accuracy (Hartree-Fock, MCSCF, valence band, DFT, variational Monte Carlo with explicitly correlated trial wave functions, fixed-node DMC, etc). Such a possibility is particularly interesting to get new insights into the nature of the pairing and localization of electrons at these various levels of description and, particularly, to understand more deeply the role of the dynamical (Coulomb hole) and nondynamical (neardegeneracy) correlation effects. A number of applications of the EPLF to simple atomic and molecular systems have been presented and systematic comparisons with ELF have been done. The results presented illustrate that for these systems EPLF is a simple and practical tool for visualizing electronic localization. In addition, it has been shown that the EPLF and the ELF exhibit similar gross features (atomic shell structure, lone pairs, bonding regions). However, there may also be some qualitative differences, particularly in the topology of the localization function. (For $\mathrm{N}_{2}$, an infinity of maxima on a torus in the region of the triple bond with EPLF, a single maximum with ELF; for the unpaired electron of the radical $\mathrm{CH}_{3}$, a minimum for EPLF, a maximum for ELF; for $\mathrm{F}_{2}$ different stability properties between the ELF and EPLF critical points, etc.). All the results presented here have been obtained from all-electron QMC calculations, we have checked for $\mathrm{F}_{2}$ that using pseudo-potentials does not change the basic features of the EPLF in the valence region. This result is expected to be general.

Finally, let us emphasize that, although EPLF appears to have some attractive features, only the analysis of many other examples covering a much wider variety of chemical situations will allow us to assess the true ability of EPLF to be used as a general tool to investigate electron localization in molecular systems.

\section{ACKNOWLEDGMENTS}

The authors gratefully acknowledge many interesting discussions with Andreas Savin and Bernard Silvi. Numerical computations have been done using QMC-MOL, a quantum Monte Carlo program for the electronic structure calculations of atoms and molecules developed at the Laboratoire de Chimie Théorique (CNRS-Paris VI) (see, www.lct.jussieu.fr). The calculations were carried out using the IBM SP computers of the Institut du Développement et des Ressources en Informatique Scientifique (IDRIS, CNRS) and the computers of the Centre de Calcul Recherche et Réseau Jussieu (CCR, Paris 6).

${ }^{1}$ O. El Akramine, W. A. Lester, X. Krokidis, C. A. Taft, A. C. Pavao, T. C. Guimaraes, and R. Zhu, Mol. Phys. 101, 277 (2003).

${ }^{2}$ A. J. Williamson, J. C. Grossman, R. Q. Hood, A. Puzder, and G. Galli, Phys. Rev. Lett. 89, 196803 (2002).

${ }^{3}$ A. Puzder, A. J. Williamson, J. C. Grossman, and G. Galli, J. Chem. Phys. 117, 6721 (2002).

${ }^{4}$ A. Puzder, A. J. Williamson, J. C. Grossman, and G. Galli, Phys. Rev. Lett. 88, 097401 (2002).

${ }^{5}$ G. Belomoin, E. Rogozhina, J. Therrien, P. V. Braun, L. Abuhassan, M. H. Nayfeh, L. Wagner, and L. Mitas, Phys. Rev. B 65, 193406 (2002).

${ }^{6}$ C. Filippi, S. B. Healy, P. Kratzer, E. Pehlke, and M. Scheffler, Phys. Rev. Lett. 89, 166102 (2002).

${ }^{7}$ A. J. Williamson, R. Q. Hood, and J. C. Grossman, Phys. Rev. Lett. 87, 246406 (2001).

${ }^{8}$ Electron Distributions and Chemical Bonds, edited by P. Coppens and M. B. Hall (Plenum, New York, 1982).

${ }^{9}$ R. F. W. Bader, Atoms in Molecules: A Quantum Theory (Clarendon, Oxford, 1990).

${ }^{10}$ S. R. Gadre, S. A. Kulkarni, and I. H. Shrivastava, J. Chem. Phys. 96, 5253 (1992).

${ }^{11}$ A. D. Becke and K. E. Edgecombe, J. Chem. Phys. 92, 5397 (1990).

${ }^{12}$ B. Silvi and A. Savin, Nature (London) 371, 683 (1994).

${ }^{13}$ B. L. Hammond, W. A. Lester, Jr., and P. J. Reynolds, Monte Carlo Methods in Ab Initio Quantum Chemistry (World Scientific, Singapore, 1994), Vol. 1.

${ }^{14}$ K. E. Schmidt and J. W. Moskowitz, J. Chem. Phys. 93, 4172 (1990).

${ }^{15}$ R. Assaraf and M. Caffarel, J. Chem. Phys. 113, 4028 (2000).

${ }^{16}$ C. J. Umrigar, K. G. Wilson, and J. W. Wilkins, Phys. Rev. Lett. 60, 1719 (1988).

${ }^{17}$ H. J. Flad, M. Caffarel, and A. Savin, Recent Advances in Quantum Monte Carlo Methods (World Scientific, Singapore, 1997).

${ }^{18}$ K. S. Liu, M. H. Kalos, and G. V. Chester, Phys. Rev. A 10, 303308 (1974).

${ }^{19}$ M. Caffarel and P. Claverie, J. Chem. Phys. 88, 1088 (1988).

${ }^{20}$ J. Casulleras and J. Boronat, Phys. Rev. B 52, 3654 (1995).

${ }^{21}$ A. Savin (unpublished).

${ }^{22}$ B. Silvi, J. Phys. Chem. A 107, 3081 (2003).

${ }^{23}$ M. W. Schmidt, K. K. Baldridge, J. A. Boatz et al., J. Comput. Chem. 14, 1347 (1993)

${ }^{24}$ T. H. Dunning, J. Chem. Phys. 90, 1007 (1989).

${ }^{25}$ T. Koga, H. Tatewaki, and A. J. Thakkar, Phys. Rev. A 47, 4510 (1993).

${ }^{26}$ M. J. Frisch, G. W. Trucks, H. B. Schlegel et al., GAussian 98, Revision A.9, Gaussian, Inc., Pittsburgh, PA, 1998.

${ }^{27}$ S. Noury, X. Krokidis, F. Fuster, and B. Silvi, TOPMOD package, Univ. Pierre et Marie Curie and CNRS, www.lct.jussieu.fr, 1997.

${ }^{28}$ R. Llusar, A. Beltràn, J. Andrés, S. Noury, and B. Silvi, J. Comput. Chem. 20, 1517 (1999).

${ }^{29}$ P. C. Hiberty, J. P. Flament, and E. Noizet, Chem. Phys. Lett. 189, 259 (1992). 
${ }^{30}$ P. C. Hiberty, S. Humbel, C. P. Byrman, and J. H. van Lenthe, J. Chem. Phys. 101, 5969 (1994).

${ }^{31}$ P. Pyykko, D. Sundholm, and L. Laaksonen, Mol. Phys. 60, 597 (1987).

${ }^{32}$ M. W. Chase, Jr., C. A. Davies, J. R. Downey, Jr., D. J. Frurip, R. A.
McDonald, and A. N. Syverud, J. Phys. Chem. Ref. Data Supp. 14, 1 (1985).

${ }^{33}$ J. M. L. Martin, J. Mol. Struct. 135, 398 (1997).

${ }^{34}$ P. Flkiger, H. P. Lthi, S. Portmann, and J. Weber, MOLEKEL 4.0 (Swiss Center for Scientific Computing, Manno, Switzerland, 2000). 